\title{
Rezensionen
}

\section{Die altpolnische Eulenspiegel-Literatur im Fokus europäischer Forschung zur zivilisatorischen Wende vom 16. zum 17. Jahrhundert}

\author{
Witold Wojtowicz: Studien zur ,, bürgerlichen Literatur" um die Wende vom \\ 16. zum 17. Jahrhundert. Peter Lang Internationaler Verlag der Wissenschaf- \\ ten, Frankfurt am Main 2015, 485 S.
}

DOI: $10.19195 / 0435-5865.144 .23$

Der vorliegende Studienband ist eine überarbeitete Fassung der polnischen Habilitationsschrift von 2010, die mit einem Grant im Nationalprogramm für die Entwicklung der Humanistik durch das Polnische Ministerium für Wissenschaft und Hochschulwesen finanziert wurde. In der deutschen Version wurden vor allem komparatistische Ausführungen und Reflexionen vertieft, wobei mehr Bezug auf die deutschsprachige Forschungslage zur bürgerlichen Literatur der Frühen Neuzeit genommen wurde. Der Band hat eine klare, überschaubare Struktur und besteht aus drei Teilen, wovon die beiden ersten theoretisch-literaturhistorisch und der letzte textbezogen analytisch-interpretatorisch ausgerichtet sind. Der Gegenstand der Studie wird in der Einleitung als „Untersuchung von kulturellen und literarischen Konventionen in der sogenannten Eulenspiegelliteratur, die ungefähr zwischen 1580 und 1650 im Königreich Polen-Litauen gedruckt wurde“ (S. 11.) festgelegt und zwar mit dem Ziel, die Entwicklung dieser Literatur in den europäischen Kontext der Zivilisationsprozesse zu stellen und zu reflektieren. Damit wird die Konventionalität als Interpretament polnischer Eulenspiegel-Literatur an der Wende zum 17. Jahrhundert gemacht. Meine ersten Kontroversen betreffen die verwendete Fachterminologie: bürgerliche, plebejische und Eulenspiegel-Literatur. Wojtowicz schreibt, jedoch nicht immer konsequent, diese Begriffe in Anführungszeichen, um deren kontroversen Charakter deutlich zu machen. Zwar ist der Begriff der bürgerlichen Literatur für das 16. und 17. Jahrhundert in der polnischen Literaturwissenschaft umstritten (S. 13), doch findet man ihn überall in literaturhistorischen Lexika und Epochensynthesen, um hier nur auf die mir vorliegenden zu verweisen. ${ }^{1}$ In den deutschsprachigen literaturhistorischen Nachschlagewerken sucht man nach diesen Begriffen vergeblich. Gleichwohl werden Termini wie bürgerliche oder städtische ggf. städtisch-bürgerliche Literatur in der Mittelalter- und Frühneuzeitforschung etwa seit den 80er Jahren gebraucht, sie verursachen jedoch eine rege

1 Jakubowski, Jan Zygmunt (Hrsg.) (1974): Literatura polska od średniowiecza do pozytywizmu. Warszawa; Hernas, Czeslaw (1980): Barok. Warszawa. 
und bis heute andauernde Diskussion. ${ }^{2}$ Wojtowicz 'Ausführungen zur polnischen „bürgerlichen Literatur" weisen viele Anschlussmöglichkeiten an diese deutsche Diskussion auf. Auch er hebt hervor, dass die Soziogenese der Autoren dieser Literatur kein Definitionskriterium sein kann, weil sie alle gebildet und oft adliger Herkunft waren. Des weiteren thematisiert diese Literatur an der Wende zum 17. Jahrhundert weder explizit Themen des städtischen Lebens noch vermittelt sie eine „bürgerliche“ Ideologie und Moralvorstellungen. ${ }^{3}$ Wojtowicz verwendet zwar den Begriff „,bürgerliche Literatur“ „als eine rein literaturwissenschaftliche konventionelle Konstruktion, welche eine gewisse Werkgruppe umfasst, die sich in den Ausgaben von Karol Badecki (1925-1950) (und den Ausgaben der gleichen Texte von jüngeren Herausgebern) befinden"(S. 11 f.), trennt aber nicht eindeutig die bürgerliche, plebejische und Eulenspiegel-Literatur ab. Das kann wirkungsästhetische Folgen haben, denn die deutsche Literaturwissenschaft benutzt die Bezeichnung plebejische Literatur nicht. Wichtig ist in diesem Kontext das Kap. 2 seiner Studie, wo der Autor auf die Struktur altpolnischer Städte und ihre Spezifik eingeht und konkludiert, dass „die etwas andere Position der Städte und des Bürgertums sowohl in der politischen Struktur der polnischen Adelsrepublik als auch in den kulturell-politischen Bestrebungen dieser Gesellschaftsschicht [...] nicht zur Bildung eines einheitlichen Standesbewusstseins und nicht zu der Herausbildung einer aktiven politischen Kultur [führte]“ (S. 15). Der Anschluss an die deutschsprachige und weiter europäische Diskussion über die Volkskultur und frühneuzeitliche Unterhaltungsliteratur wird auch durch einen Methodenpluralismus erreicht, den ich hier besonders hervorheben will. Die europäische literaturwissenschaftliche Frühneuzeitforschung profitiert bereits seit den 70er Jahren von den Ergebnissen der historischen und soziologischen Forschung und eignete sich später auch innovative Perspektiven der historischen Anthropologie und der Kulturwissenschaften an. Diese interdisziplinäre Erweiterung des Untersuchungsfeldes und seiner Semantik wurde schnell in der polnischen Germanistik für die Barockforschung mit den deutschsprachigen Texten übernommen, ${ }^{4}$ während die Polonistik fast ausschließlich angelsächsische Forschungsliteratur rezipierte. Die Studie von Wojtowicz erweitert den Polonisten diesen Zugang zur europäischen Forschung auch mit deutschen Publikationen, vor allem den Arbeiten von Wilhelm Röcke und Hans Rudolf Velten (2005). Für die Thematisierung der Populärkultur in der polnischen Adelsrepublik war für ihn die Studie von Peter Burke (1981) mit seiner Definition der Volkskultur als ,eine nicht-elitäre Kultur, als eine Kultur der Klassen der Unterlegenen innerhalb einer Kulturhegemonie“" ausschlaggebend. Wojtowicz unterstreicht, dass die polnische „bürgerliche Literatur Stereotypen der Adelskultur [wieder-

2 Als programmatisch für diese Diskussion und die Klärung der Begriffe gilt der Beitrag von Kurt Ruh „Versuch einer Begriffsbestimmung von „städtischer Literatur“ im deutschen Spätmittelalter“. In: Fleckenstein, Josef u. Stackmann, Karl (1989) (Hrsg.): Über Bürger, Stadt und städtische Literatur im Spätmittelalter. Bericht über Kolloquien der Kommission zur Erforschung der Kultur des Spätmittelalters 1957-1977. Göttingen. Vgl. auch: Kirschner, Carola (1996): Hermen Bote. Städtische Literatur um 1500 zwischen Tradition und Innovation. Duisburg; Peters, Ursula (1983): Literatur in der Stadt. Studien zu den sozialen Voraussetzungen und kulturellen Organisationsformen städtischer Literatur im 13. und 14. Jahrhundert. Tübingen.

3 Vgl. dazu Schnell, Rüdiger (1978): Zum Verhältnis von hoch- und spätmittelalterlicher Literatur. Versuch einer Kritik. Berlin.

${ }^{4}$ Vgl. Studien der Breslauer Barockforscher, wie Mirosława Czarnecka, Jolanta Szafarz, Kalina Mróz-Jabłecka, Tomasz Jabłecki. 
holt]“ (S. 49) und „,von Konventionen, Motiven und Relikten der Hof- und Adel-Literatur durchdrungen“" war (S. 85). Er sieht dabei ein, im Einvernehmen mit Burke, dass es doch richtiger wäre, statt von einer Kluft zwischen der hohen und niederen Kultur, von einer „gegenseitigen Durchdringung oder Verbindungen zwischen der Gelehrtenkultur und der Populärkultur, zwischen dem Wissen der vor allem auf mündlicher Überlieferung basierten Agrargesellschaft und der das Schreiben beherrschenden Gelehrtenkultur stattgefunden hat" (S. 57) zu sprechen. Als Beispiel für dieses kulturelle Phänomen der Annektierung von Konventionen hoher Kultur durch die Populärkultur gibt Wojtowicz den Text „Peregrynacja dziadowska“ (Bettlers Reise) des Eulenspiegel-Autors Januarius Sowizralius von 1612 an. Erst um die Mitte des 17. Jahrhunderts wird in der polnischen Kultur eine völlige Trennung zwischen Volkskultur und Elite-Kultur sichtbar, wobei mit Elite nicht der polnische Landadel, der rückständig und Neuem gegenüber misstrauisch war, sondern die Magnaten-Höfe gemeint sind. Die Veränderungen der Verhaltensregeln infolge der Zivilisationsprozesse gingen im frühneuzeitlichen Europa nur langsam voran. In Polen konnten sie erst um 1800 zur Geltung kommen als sich zur Zeit des Großen Sejm 1788-1792 der eigenständige Bürgerstand herauskristallisieren konnte.

Teil II der Studie wird den Epochendiskursen der Zivilisation und Gewalt gewidmet. Dabei rekapituliert Wojtowicz sehr umfangreich und in meiner Einschätzung unnötig, das Werk des Soziologen Norbert Elias (1995). An den deutschen Universitäten ist es für die Geisteswissenschaften eine Pflichtlektüre und es liegt auch auf Polnisch sowohl in Auswahl als auch in vollem Umfang vor. Der III. Teil des Bandes konzentriert sich auf den Lachdiskurs und den Karneval als Ventil für die feudale Gesellschaft, die Normen des Staates anzuerkennen. In der Diskussion der ars iocandi kommt Michael Bachtin (1969 u. 1995) die vorherrschende Position zu. Ich finde diesen Teil der Studie besonders interessant, obwohl das deskriptive Kapitel zu Bachtins performativen Lachdiskurs auch unnötig weit ausgebaut wurde. Besonders aufschlussreich für das Ziel dieser Studie scheinen die beiden Exkurse zu sein: 1. Über das Lachen in Łukasz Górnicki „Dworzanin polski“, der Übersetzung und Bearbeitung des „Hofmanns“ Castigliones und 2. Über die Babiner Republik, die als kulturelles Phänomen der verkehrten Welt bereits seit der 2. Hälfte des 16. Jahrhunderts an die europäische karnevaleske Tradition anknüpfen konnte und ein Beispiel der gelehrten Lachgemeinschaft darstellte. Für mein Interesse an den Texten der polnischen Literatur des 17. Jahrhunderts im Fokus genderorientierter, anthropologischer Untersuchungen, sind Kapitel 10 und 11 von besonderem Interesse. Wojtowicz widmet hier seine Aufmerksamkeit dem misogynen Geschlechterdiskurs in der altpolnischen Kultur, der vor allem in den Texten des Frauenparlaments dominierte. Die polnische Querelle des femmes in der Frühen Neuzeit war bescheiden, eigentlich nur ein schwaches Echo der westeuropäischen Debatte, und lässt sich mit der wahren Flut von frauenschmähenden Schriften in Deutschland gar nicht vergleichen. ${ }^{5}$ Leider bezieht Wojtowicz in seinen Analysen und Interpretationen weder die polnische noch die umfangreiche europäische Literatur zur Querelle des femmes ${ }^{6}$ mit ein. Interessante Ausführungen, kontextuelle Verweise und Interpretationen dieser Textgruppe „Sejmy niewieście“ (Frauenparlaments), könnte man sicherlich durch die Einbeziehung der multimedialen Tradition des Kampfes um die Hose noch profunder machen. Die reiche Ikonographie sowohl zur karnevalesken Verkehrung der Geschlechterhierarchie als auch zu

5 Vgl. Bogucka, Maria (1998): Białogłowa w dawnej Polsce. Warszawa.

${ }^{6}$ Vgl. Jahrbuch für Frauenforschung (1997): Die europäische Querelle des Femmes. Geschlechterdebatten seit dem 15. Jahrhundert. Hg. v. Gisela Bock u. Margarete Zimmermann. 
Disziplinierungsmaßnamen, die Nathalie Zemon Davies (1987) als Riten der Gewalt beschrieben hatte, könnten dann die Thematisierung dieser Rollenumkehrung in Verbindung mit Macht-, Bildungs- und Staatsrechtsdiskursen viel aufschlussreicher in den europäischen Kontext stellen. Das gilt auch für die Ehe- und Altersdiskurse, die bei Wojtowicz ausschließlich mit Karneval und ars iocandi thematisiert werden. In der deutschen Literatur ist die Figur der alten Jungfer seit dem 15. Jahrhundert nachweisbar, und zwar nicht im Kontext des Karnevals, sondern primär im Diskurs der ungeratenen Ehe, also einer Vermählung, in der der Altersunterschied der Brautleute gravierend von der Norm abweicht. Sie erscheint als alte Vettel, d.h. alte, wollüstige Frau, die körperlich abstoßend wirkt trotz der Schminke und moralisch die Norm verletzt (Czarnecka 2008). Wojtowicz attestiert den polnischen satirischen Texten dieser Gruppe ausschließlich eine didaktische Funktion der Wiederherstellung der Norm und der Disziplinierung von Frauen und auch Männern. Sollte man aber diese Texte mit der sozialen Wirklichkeit konfrontieren, wie etwa mit dem Witwen- oder Ehediskurs, so könnte man diese Semantik erweitern, oder wenigstens ihren latenten oder auch sichtbaren Didaktismus hinterfragen.

Als irritierendes Defizit der besprochenen Studie sehe ich das Fehlen der Bibliographie am Ende. Eine detaillierte Zusammenfügung der primären und sekundären Literatur ist unerlässlich in wissenschaftlichen Publikationen und selbst ein Namenregister kann dieses evidente Manko nicht aufheben. Leider lässt auch die Übersetzung ins Deutsche viel zu wünschen übrig. An vielen Stellen wird durch eine wortwörtliche Übertragung aus dem Polnischen der Sinn der Ausführung unklar. Ich fasse zusammen:

Witold Wojtowicz wollte mit seinem Band die altpolnische Literatur in den Fokus der europäischen Forschung zur zivilisatorischen Wende an der Schwelle vom 16. zum 17. Jahrhundert stellen. Er konzentrierte sich auf Texte der Populärliteratur und ließ sowohl religiöses Schrifttum als auch Gebrauchsliteratur aus. In seinen interdisziplinär ausgerichteten Untersuchungen zieht er eine vergleichende Analyse vor, um auf vielschichtige Verschränkungen zwischen den einzelnen Texten zu verweisen und damit literarische Konventionen als Interpretament zu nutzen, was neue Deutungen der bekannten Texte ermöglichte. Damit schreibt sich diese Studie in die neuesten Forschungen zur Re-Vision der Epoche der Frühen Neuzeit ein. ${ }^{7}$ Es ist eine komparatistische Studie, die dank der Übersetzung der angeführten Zitate aus der polnischen Literatur den deutschen Lesern eine Annäherung an diese Texte, die ja sonst nur auf Polnisch zugänglich sind, ermöglicht. Die Verschränkung des polnischen Forschungsstandes mit den Ergebnissen europäischer Forschung zu Nationalliteraturen zeigt sich als sehr ergiebig und aufschlussreich für weitere komparatistische Studien.

\section{Literatur}

Bogucka, Maria (1998): Białoglowa w dawnej Polsce. Warszawa.

Burke, Peter (1981): Helden, Schurken und Narren. Europäische Volkskultur in der frühen Neuzeit. Stuttgart.

${ }^{7}$ Vgl. Höfele, Andreas / Müller, Jan-Dirk / Oesterreicher, Wulf (2013) (Hrsg.): Die Frühe Neuzeit. Revisionen einer Epoche. Berlin. 
Czarnecka, Miroslawa (2008): Misogyne Lachgemeinschaft. Barocke Frauensatire im deutsch-polnischen Vergleich. In: Arend, Stefanie e.a. (Hrsg.): Anthropologie und Medialität des Komischen im 17. Jahrhundert (1580-1730) (= Chloe 40). Berlin. S. 357-370.

Elias, Norbert (1995): Über den Prozess der Zivilisation. Soziogenetische und psychogenetische Untersuchungen. 2 Bde. Amsterdam.

Röcke, Wilhelm / Velten, Hans Rudolf (2005) (Hrsg.): Lachgemeinschaften. Kulturelle Inszenierungen und soziale Wirkungen von Gelächter im Mittelalter und in der Frühen Neuzeit. Berlin/ New York.

Zemno Davies, Nathalie (1987): Humanismus, Narrenherrschaft und die Riten der Gewalt. Gesellschaft und Kultur im frühneuzeitlichen Frankreich. Frankfurt am Main.

Mirosława Czarnecka

(Universität Wrocław)

ORCID: 0000-0001-5226-5260

Mirosława Czarnecka, Uniwersytet Wrocławski, Instytut Filologii Germańskiej, Pl. Nankiera 15b, 50-140 Wrocław, Polen, E-Mail: miroslawa.czarnecka@edu.uni.wroc.pl

Received: 12.08.2018, accepted: 8.04.2019

\section{Georg Rudolf Weckherlin (1584-1653): neue Forschungsperspektiven und Zugänge zum Werk des vergessenen Dichters}

Heiko Ullrich (Hrsg.): Privatmann - Protestant - Patriot - Panegyriker Petrarkist - Poet: Neue Studien zu Leben und Werk Georg Rudolf Weckherlins (1584-1653). Ralf Schuster Verlag, Passau 2018, 442 S.

DOI: $10.19195 / 0435-5865.144 .24$

Heiko Ullrich präzisierte das Ziel seines Bandes als eine neue Öffnung des Forschungsinteresses für Weckherlin durch „die Präsentation einer Fülle von Wegen [...], auf denen die germanistische Literaturwissenschaft bei der Erforschung des lange vernachlässigten Dichters [...] voranschreiten könnte.“ (S. 1) Zu diesem Zweck sind die einzelnen Beiträge einer Typologie der identitätsstiftenden Rollen unterstellt: Weckherlin wird als Privatmann, Protestant, Patriot, Panegyriker, Petrarkist und Poet vorgestellt. Schon diese bereits auf dem Titelblatt formulierte Typologie lässt einen doppelten Fokus erkennen: Weckherlin wird zunächst von der Biographie her diskutiert und dann werden wichtige thematische, gattungsgeschichtliche und motivische Schwerpunkte seines Werks analysiert. Neue Perspektiven und Zugänge zum Werk des Dichters ergeben sich aus der anthropologischen, lebensweltlichen, genderorientierten, poetologischen und wirkungsästhetischen Kontextualisierung seiner Situation als Dichter im Exil, der ohne direkten Bezug zur deutschen Respublica litteraria und zu den kulturtragenden Institutionen in der Heimat stand. In der europäischen Metropole London wirkte und schrieb er mit anderen Erfahrungen als die 